EESTI NSV TEADUSTE AKADEEMIA TOIMETISED. XIV KOIDE FOOSIKA-MATEMAATIKA-JA TEHNIKATEADUSTE SEERIA. 1965, NR. 2

ИЗВЕСТИЯ АКАДЕМИИ НАУК ЭСТОНСКОИ ССР. ТОМ ХІV

СЕРИЯ ФИЗИКО-МАТЕМАТИЧЕСКИХ И ТЕХНИЧЕСКИХ НАУК. 1965, № 2

Э. ЛИППМАА, П. ЛУИГА

\title{
О НЕРАВНОМЕРНОСТИ СКОРОСТИ ПОТОКА ГАЗА В ПРЕПАРАТИВНОЙ ГАЗОВОЙ ХРОМАТОГРАФИИ
}

Для достижения требуемой пропускной способности в препаративной газовой хроматографии необходимо применять колонки большого диаметра. Однако увеличенне диаметра колонки свыше 15-25 мм приводит к резкому снижению ее эффективности $\left[{ }^{1-4}\right]$.

В ходе анализа причин падения эффективности при увеличении диаметра хроматографических колонок целесообразно исходить из уравнения ван Деемтера [5] с уточнениями Джонса и Дал Ногаре $\left[{ }^{6,7}\right]$

$$
H=A+\frac{B}{u}+C_{l} u+C_{g} u,
$$

где $H$ - высота теоретической тарелки;

$A$ - вихревая диффузия;

$B$ - продольная (молекулярная) диффузия;

$C_{l}$ - внутренняя (жидкофазная) диффузия;

$C_{g}$ - внешняя поперечная (газофазная) диффузия;

$u$ - скорость газа.

Приведенное уравнение только приближенно описывает хроматографическое разделение. Есть серьезные основания считать член $A$ артефактом $\left[{ }^{8,9}\right]$, зависящим главным образом от способа ввода пробы $\left[^{10}\right]$ и, кроме того, в уравнении отсутствуют поправки на перепад давления в колонке [11, 12].

Из приведенных членов только $C_{g} u$ и $A$ могут зависеть от радиуса колонки $R_{0}$. Член $A$ всегда мал и подчас даже отсутствует [ ${ }^{11}$ ], поэтому в дальнейшем будем рассматривать только $C_{g} u$. Ван Деемтер считал $C_{g} u$ малым в сравнении с $C_{l} u$, но впоследствни выяснилось, что это неправильно $\left[{ }^{13-16}\right]$ и что этот показатель может иметь большое значение $[17-20]$.

Целесообразно рассмотреть $C_{g} u$ в развернутом виде:

$$
C_{g} u=C_{1}{ }^{\prime} u+C_{1}{ }^{\prime \prime} u+C_{2} u+C_{3} u,
$$

где $C_{1}^{\prime}-$ внешняя диффузия между зернами;

$C_{1}^{\prime \prime}$ - внешняя диффузия в зернах $\left[{ }^{17}\right]$;

$C_{2}$ - распределение скоростей газа в колонке;

$C_{3}$ - корреляция между $C_{1}$ и $C_{2}\left[{ }^{6}\right]$. 
Корреляцию между $C_{l}$ и $C_{1}\left[{ }^{17}\right]$ считаем малой. Зависимость от условий опыта подробно исследована Голеем $\left[{ }^{6,20}\right]$. Он исходил из параболического распределения скоростей в капилляре и назвал

$$
\left(C_{1}^{\prime}+C_{2}+C_{3}\right)
$$

динамической диффузией.

В колонках с зернистым наполнением положение сложнее. Здесь может наблюдаться зависимость скорости газа от радиуса как в отдельных капиллярах между зернами, так и в колонке в целом. Из опытных данных $\left[{ }^{21}\right]$ и теоретических соображений $\left[{ }^{10}\right]$ следует, что первый эффект не имеет практического значения. Существенное значение имеют внешняя диффузия в зернах $\mathrm{C}_{1}^{\prime \prime}\left[{ }^{17}\right]$ и взаимодействие отдельных каналов течения в пористой среде $\left[{ }^{12,19}\right]$, что практически совпадает с членом $C_{2}$. Для вычисления или измерения $C_{3}$ нет никаких возможностей. Известно только [6], что

$$
C_{3} \ll C_{1}^{\prime}+C_{2} \text {. }
$$

С точки зрения препаративной газовой хроматографии самое существенное значение имеет член $C_{2} u$, который и является главной причиной снижения эффективности хроматографических колонок при увеличении диаметра. Влияние неравномерностей скорости газа в препаративной колонке рассмотрено Голеем [3], который отдельно проанализировал влияние повышенной скорости газа у стенок колонки (стеночный эффект) и влияние макроскопической неравномерности течения. Обзор различных факторов, приводящих к снижению эффективности, составлен Джиддинсом $\left.{ }^{12}\right]$. Конкретные формулы, составленные различными исследователями, могут быть представлены в форме

$$
{ }_{\Delta} H=f\left(\alpha, \frac{R}{R_{0}}, u\right) \frac{R_{0}^{2}}{D_{g}} u,
$$

где $\alpha-$ полярный угол;

$R-$ радиус;

$R_{0}$ - радиус колонки, отдельного капилляра или зерна;

$u$ - средняя скорость газа;

$D_{g}$ - коэффициент газофазной диффузии.

Структура функции $f\left(\alpha, \frac{R}{R_{0}}, u\right)$ зависит от допущений, сделанных о характере потока в колонке. Первые два уравнения на нижеследующей схеме описывают стеночный эффект. По-видимому, правильнее связывать завышенную скорость потока у стенок с радиусом зерна [ $\left.{ }^{3}\right]$, чем с радиусом колонки $\left[{ }^{22}\right]$, так как градиент скорости, вызванный стеночным эффектом, зависит главным образом от пристенного слоя, толщина которого мало превышает диаметр зерна. Стеночный эффект наблюдается во всех адсорберах, скрубберах и хорошо исследован. Скорость газа у стенок во много раз превышает среднюю скорость [25, 26]. Однако, по другим данным, у стенок может наблюдаться и замедленное течение $\left[{ }^{27},{ }^{28}\right]$. Чисто эмпирическая формула Хупе $\left[{ }^{29}\right]$ тоже позволяет оценивать величину стеночного эффекта при различных скоростях газа.

Последние три уравнения $[3,23,24]$ описывают влияние неравномерного распределения скоростей по сечению колонки и основаны на опытах Хайтена $\left[{ }^{23}\right]$. Хайтен нашел в препаративных колонках диаметром $7,6 \mathrm{~cm}$ приблизительно параболическое распределение скорости газа с максимальной скоростью у стенок. О действительном распределении скорости газа 


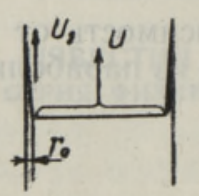

$$
{ }_{\Delta} H=(4,5 \div 18) \frac{r_{0}^{2}}{D_{g}} u \quad \text { Голей }\left[{ }^{3}\right]
$$

$\left|t_{R_{0}}^{u_{1}^{u}}\right|$

${ }_{\Delta} H=\left(\frac{\frac{u_{1}}{u}-1}{\frac{2 \delta}{R_{0}} \frac{u_{1}}{u}+1}\right)^{2} \frac{R_{0}^{2}}{D_{g}} u \begin{aligned} & \text { Алексеева, } \\ & \text { Жуховицкий, } \\ & \text { Туркельтауб }\left[{ }^{22}\right]\end{aligned}$

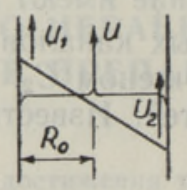

$$
{ }_{\Delta} H=\left(\frac{u_{1}}{u_{2}}-1\right)^{2} \frac{4 R_{0}^{2}}{D_{g}} u \quad \text { Голей }\left[{ }^{3}\right]
$$

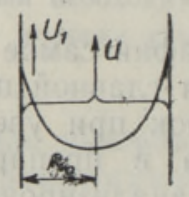

$$
{ }_{\Delta} H=2 k \frac{R_{0}^{2}}{D_{g}} u
$$

Хайтен $\left[{ }^{23}\right]$

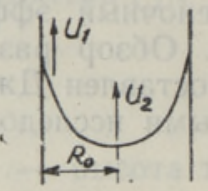

${ }_{\Delta} H=\left(\frac{u_{1}-i_{2}}{u_{1}+u_{2}}\right)^{2} \frac{1}{24 \gamma} \frac{R_{0}^{2}}{D_{g}} u$ Джиддингс [24]

в колонках очень мало данных. Обычно считают, что снижение эффективности препаративных колонок связано со стеночным эффектом (повышенной скоростью газа у стенок), но в вопросе нет ясности. Сказанное прекрасно иллюстрируется результатами дискуссии, проведенной в Мичиганском университете [30].

\section{Экспериментальная часть}

Для определения распределения скоростей газа при выходе из сорбента применялись электрические анемометры с нагретой проволокой [25] и химические методы $[23,31,32]$. Следует, однако, отметить, что как первый, так и второй методы имеют большие недостатки. Чувствительность и стабильность электрических анемометров недостаточна для измерения малых разностей $(<1$ см/сек) скорости газа. Химические методы позволяют определить форму фронта, но не дают возможности исследовать формы замыкающего края полосы. Мы применяли большое количество (42 и 30) упрощенных пламенных детекторов для определения углеводородного компонента в газе-носителе, (азот или водород) по окрашиванию в яркий цвет бесцветного водородного микропламени.

Для опытов применяли препаративные колонки диаметром 75 мм и длиной $154 \mathrm{~cm}$, а также диаметром 32 мм и длиной $285 \mathrm{~cm}$, наполненные диатомитовым кирпичом (зернение от 0,25 до 0,5 ), пропитанным дибутилфталатом (30\% от веса кирпича) и наполненные диатомитовым кирпичом без жидкой фазы (зернение от 0,25 до 0,5 мм). Некоторые опыты были проведены с пустыми трубками. В колонку диаметром 75 мм с конца были введены капилляры внешним диаметром 0,8 мм. Отверстия капил- 


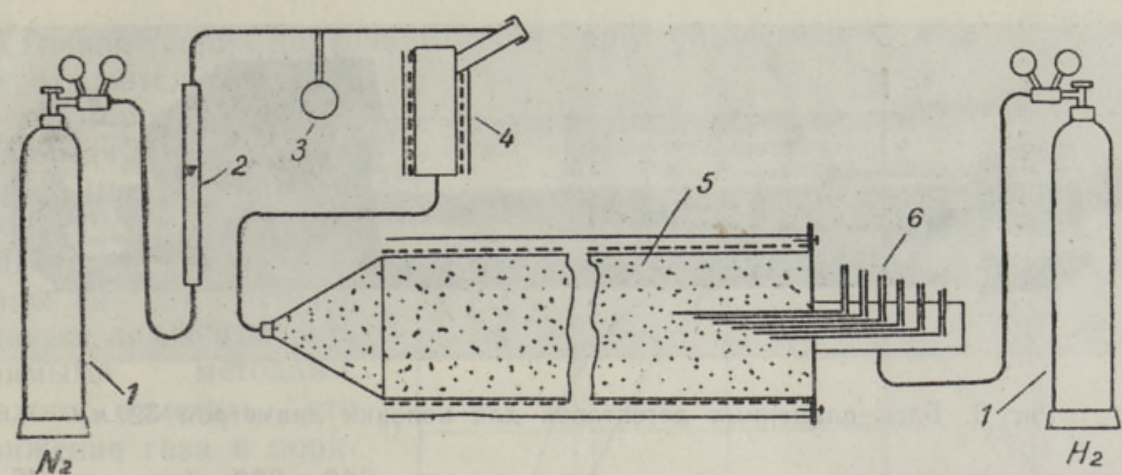

Рис. 1. Схема установки для исследования стеночного эффекта, 1 - баллон, 2 - ротаметр, 3 - манометр, 4 - испаритель, 5 - колонка, 6 - пламенные детекторы,

ляров в колонке, а также микрогорелки на другом конце вне колонны были размещены в шахматном порядке с шагом 10 мм. K каждой микрогорелке подводили вспомогательным капилляром водород. Длина пламени чистого водорода поддерживалась на уровене 5 мм. В колонку диаметром 32 мм сбоку до середины трубки были введены капилляры диаметром 0,4 мм, тоже в шахматном порядке с шагом 7 мм. К внешним концам капилляров подводили водород. Общая схема установки приведена на рис. 1 , конструкции капиллярных систем - на рис. 2 и 3. Распределение светящегося пламени точно воспроизводило распределение концентрации углеводорода (н-гептан) в хроматографической полосе.

Результаты одного из опытов приведены на рис. 4.

Оказывается, что скорость передвижения фронта максимальна в середине колонки; у замыкающего края полосы наблюдаются даже длинные «хвосты» вдоль стенок (рис. 5). Эти результаты наблюдались в большей или меньшей мере во всех опытах. Разность скоростей в середине и

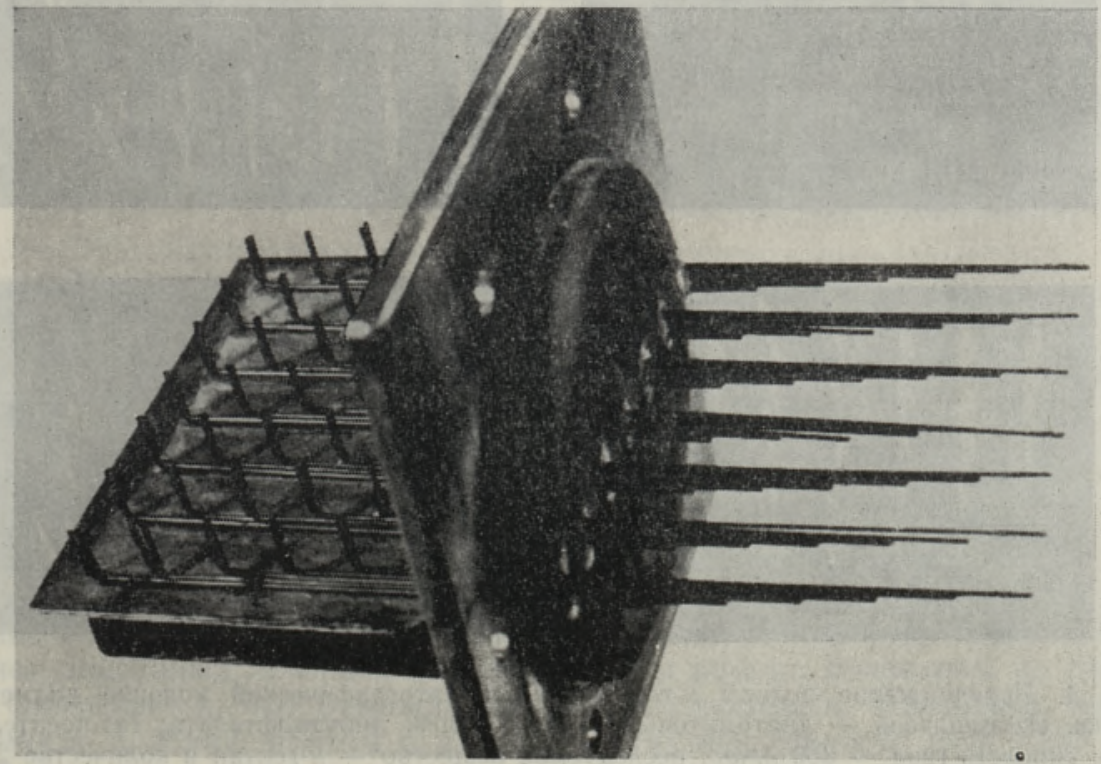

Рис. 2. Блок пламенных детекторов для колонки диаметром 75 мм. 


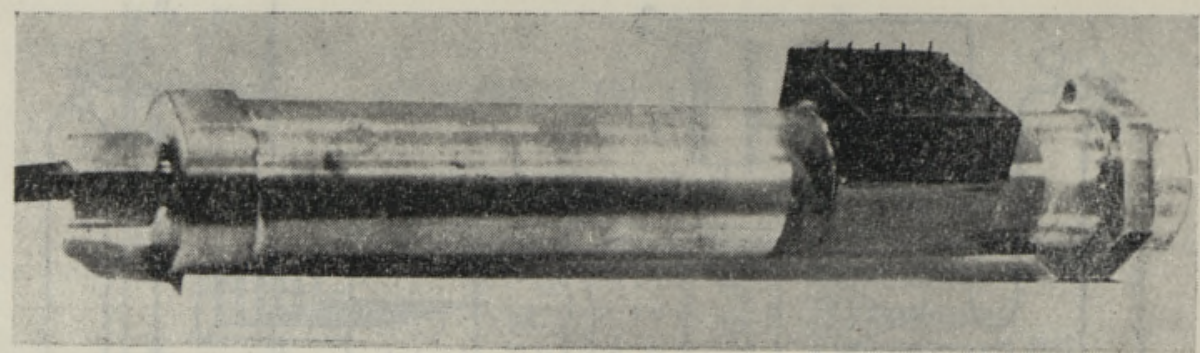

Рис. 3. Блок пламенных детекторов для колонки диаметром 32 мм.

на периферии максимальна при скоростях газа 200-600 л/час для 75 мм колонки, что примерно соответствует нормальному рабочему диапазону для препаративных разделений. При скоростях 100 и $900 \Omega /$ час разность скоростей меньше и приближается к нулю. Она больше в случае применения в качестве газа-носителя азота и значительно меньше в случае водорода, что согласуется с данными Жуховицкого и Туркельтауба [ $\left.{ }^{33}\right]$.

Большая скорость передвижения фронта в середине колонки является следствием большей скорости газа в центре, а не неравномерного распределения жидкой фазы, что могло бы вызвать точно такой же эффект [24]. Это подтверждается опытами с колонкой, наполненной диатомитовым кирпичом (зернение $0,25-0,5$ мм) без жидкой фазы. Несмотря на очень малый удерживаемый объем, максимальная скорость передвижения фронта наблюдалась всегда в середине колонки.

Опыты с колонкой диаметром 32 мм дали точно такие же результаты, только абсолютная разность скоростей была меньше.
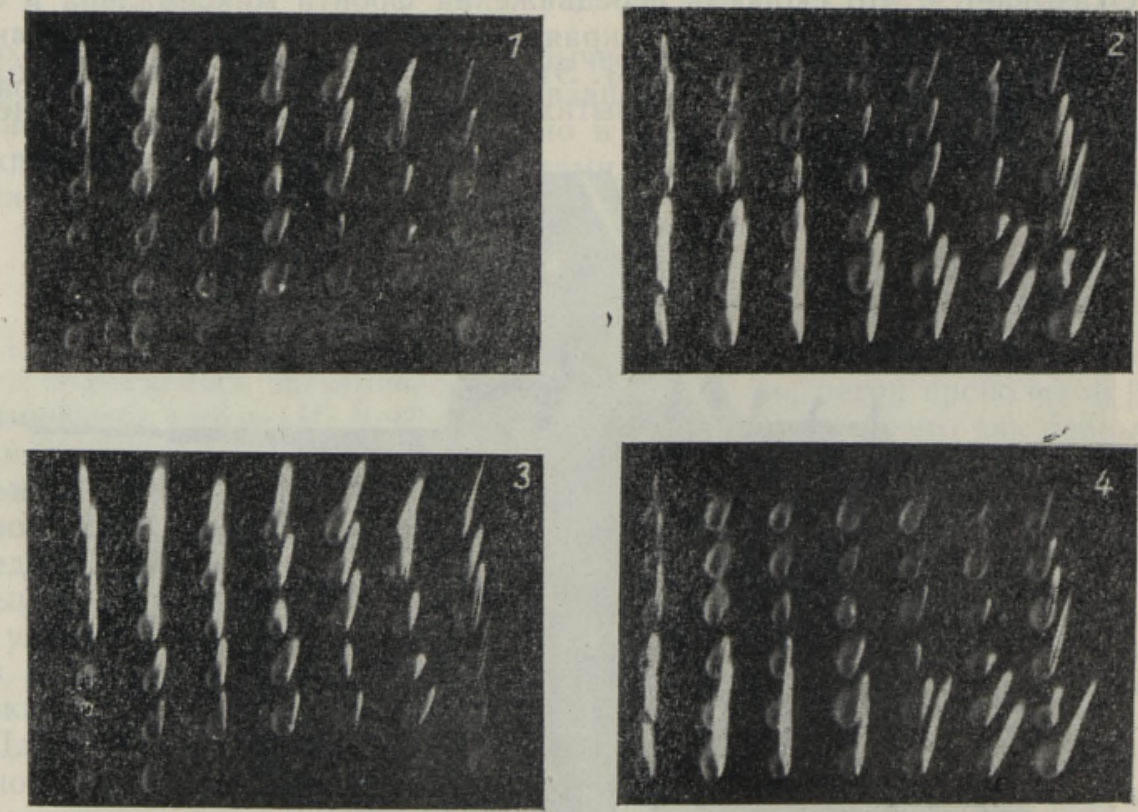

Ркс. 4. Передвижение полосы н-гептана в хроматографической колонке диаметром 75 мм. Наполнитель - диатомитовый кирпич с $30 \%$ дибутилфталата; газ-носитель азот; скорость газа - $500 \Omega /$ час; исследуемое вещество - н-гептан в количестве 2 мл; температура $-105^{\circ} \mathrm{C}$; съемка без фильтра, экспозиция - 1 сек; 1 и 3 фронт полосы, 2 и 4 - замыкающий край. 
Точная теория движения газа в пористой среде пока еще отсутствует.

Те относительные разности скорости газа, абсолютная величина которых имеет существенное значение в газовой хроматографии очень малы и сомнительно, удастся ли их измерять прямыми методами. Важно отметить, что движение газа в пористой среде ламинарно до $\mathrm{Re}=1 \div 10 \quad\left[{ }^{34}\right]$, $\operatorname{Re}=5 \div 6, \operatorname{Re}=160 \div$ $\div 170\left[{ }^{35}\right], \mathrm{Re}=40 \div 50$ $\left.{ }^{36}\right]$ и переход к турбулентному течению очень плавный. В капиллярных отверстиях препаративных колонок кри-

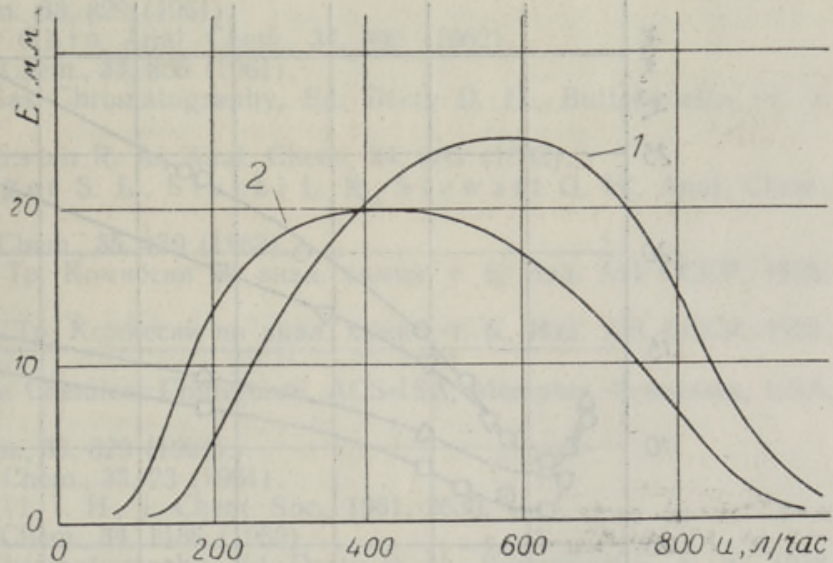

Рис. 5. Неравномерность фронта $E$ в зависимости от скорости газа-носителя $u$. Диаметр колонки -75 мм, длина - 1,54 $м$, н-гексан; насадки: $I$ - диатомитовый кирпич без жидкой фазы, 2 - диатомитовый кирпич, пропитанный $30 \%$ дибутилфталатом. терии Рейнолдса $\operatorname{Re}<1$.

В пустой трубке диаметром 75 мм и длиной 154 см фронт передвижетия паров $\boldsymbol{H}$-гептана имеет параболическую форму с максимальной скоростью в середине, как и следует из теории ламинарного течения.

В связи с вышеизложенным кажется удивительным, как могут улучшить хроматографическое разделение круги с центральным отверстием, размещенные в колонке через несколько диаметров. Такие круги применяли с хорошими результатами Фризоне $\left[{ }^{37}\right]$ и $Э$ ми $\left[{ }^{30}\right]$.

Мы проводили опыты с колонкой диаметром 75 мм и длиной $154 \mathrm{~cm}$ (наполнитель - диатомитовый кирпич 0,25-0,5 мм с $30 \%$ дибутилфталата, газ-носитель - азот, разделяемое вещество - $H$-гексан, 2 мл, температура $\left.105^{\circ} \mathrm{C}\right)$. Были применены круги из толстой бумаги диаметром центрального отверстия 12 и 32 мм, плотно прилегающие к стенкам, и круги диаметром 68 мм с малыми выступами, оставлявшими периферийный зазор 3,5 м.. В. колонку ставили во всех опытах 7 перегородок через равные расстояния. Высота теоретической тарелки вычислена по формуле

$$
H=\frac{L}{16\left(\frac{t_{R}}{b}\right)^{2}},
$$

где $L-$ длина колонки; $b$ - ширина пика; $t_{R}-$ время удерживания.

Скорость вычислена как средняя по формуле

$$
u=\frac{1}{2} u_{b}\left(1+p_{b}\right)
$$

где $u$ - средняя скорость; $u_{b}$ - скорость газа перед колонкой; $p_{b}-$ давление газа перед колонкой. Для опытов был использован хроматограф, ранее описанный авторами [38]. Результаты опытов приведены на рис. 6.

Оказывается, что бумажные круги действительно повышают эффективность колонки, притом в рабочем диапазоне скорости газа $(150-300$ л/час) весьма значительно. Но еще лучше действуют перегородки с периферийным зазором, которые задерживают движение газа в середине 


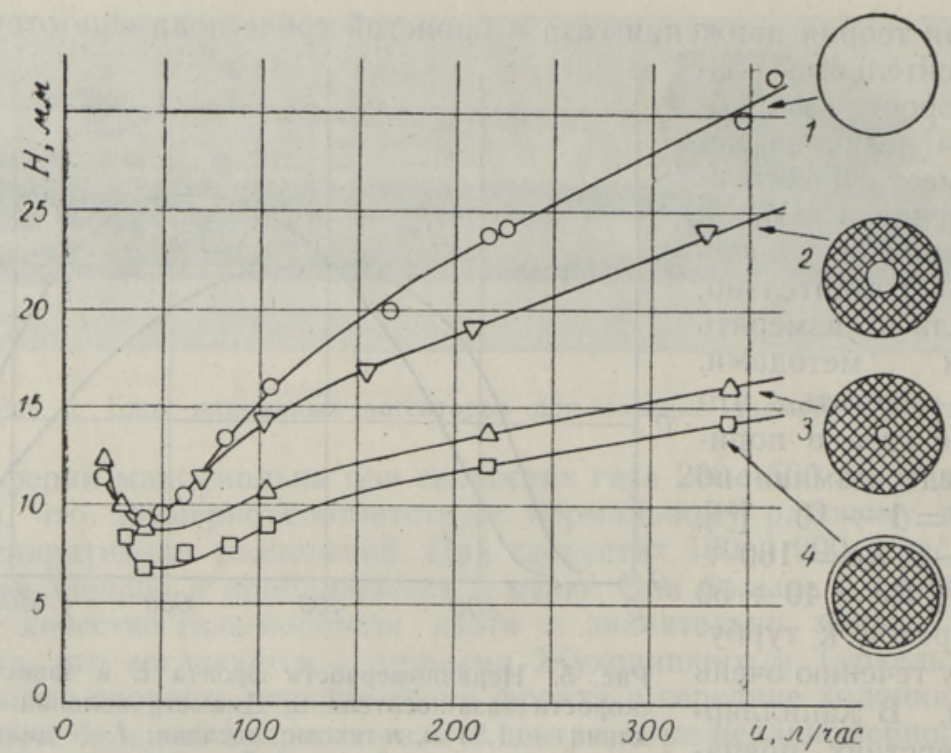

Рис. 6. Зависимость высоты теоретической тарелки $H$ от скорости газа-носителя $u$ для колонки диаметром 75 мм: 1 - колонка без перегородок; 2 - колонка с перегородками, диаметр центрального отверстия 32 мм; 3 - колонка с перегородками, диаметр центрального отверстия 12 мм; 4 - колонка с перегородками, ширина периферийного зазора $3,5 \mathrm{mм}$.

колонки. По-видимому, главной причиной увеличения эффективности колонки является перемешивание газового потока. Важность перемешивания газа в препаративных колонках подчеркивали также Голей [] и Байер [1].

\section{Выводы}

1. В исследованных колонках с зернистым наполнением истинный стеночный эффект не имеет существенного значения в определении высоты теоретической тарелки.

2. Падение эффективности препаративных колонок с увеличением диаметра связано с неравномерным распределением скорости газа вдоль всего диаметра колонки.

3. Картина распределения скоростей газа в препаративных колонках может быть различная, в зависимости от наполнителя и техники наполнения. Максимальная скорость газа может наблюдаться как в центре, так и в периферии колонки.

4. Для повышения эффективности препаративных колонок целесообразно перемешивать газовый поток в колонке или между короткими секциями в соединительных трубках.

\section{Л ИТЕ Р А Т У Р А}

1. B a y e r E., W it s c h H. G., Z. Anal. Chem., 170, 278 (1959).

2. G i d d i n g s J. C., Anal. Chem., 34, 37 (1962).

3. Gol a y M. J. E., Gas Chromatography, Ed. Noebels H. J., Wall R. F., Brenner N., Academic Press, N. Y., 1961, p. 11.

4. Johns T., B u rn ell M. R., Ca r le D. W., Gas Chromatography, Ed. Noebels H. J., Wall R. F., Brenner N., Academic Press, N. Y., 1961, p. 207.

5. van Deemter I. I., $Z$ ui derweg F. I., K li nkenberg A., Chem. Eng. Sci,, 5, 271 (1956). 
6. J o n e s W. L., Anal. Chem., 33, 829 (1961).

7. Da I Nogare S., Jen Chi u, Anal. Chem., 34, 890 (1962).

8. K i e s e l b a c h R., Anal. Chem., 33, 806 (1961).

9. Little w o od A. B., Gas Chromatography, Ed. Desty D. H., Butterworths, N. Y. 1958 , p. 23.

10. Gi d d ing s I. C., R o b is o n R. A., Anal. Chem., 34, 885 (1962).

11. G i d d i n g s I. C., S e a ger S. L., S t u ck i L. R., S t ew a rt G. H., Anal. Chem., $32,867(1960)$.

12. G i d d i n g s I. C., Anal. Chem., 35, 439 (1963).

13. Ж ухо виц ки й А. А., Тр. Комиссии по анал. химии, т. 6, Изд. АН СССР, 1955, стр. 33 .

14. Т уркельт а уб Н. М., Тр. Комиссии по анал. химии, т. 6., Изд. АН СССР, 1955 , стр. 146.

15. J o nes W. L., Southwide Chemical Conference, ACS-ISA, Memphis, Tennessee, USA, Dec. 1956.

16. J o n e s W. L., Anal. Chem., 33, 829 (1961).

17. K i e s e l b a ch R., Anal. Chem., 33, 23 (1961).

18. B ohemen I., Purnell I. H., J. Chem. Soc., 1961, 2630.

19. G i d d i n g s I. C., Anal. Chem., 34, 1186 (1962).

20. Gol a y M. I. E., Gas Chromatography, Ed. Desty D. H., Butterworths, N. Y., 1958, p. 36 .

21. Perrett R. H., Purnell I. H., Anal. Chem., 35, 430 (1963).

22. Алексеев а К. В., Ж уховицкий А. А., Туркельтауб Н. М., Хим. и техн. топлива и масел, 4, 60 (1962).

23. Huyten T. H., van Beersum W., Rijnders G. W. A., Gas Chromatography, Ed. Scott, R. P. W., Butterworths, Washington 1960, p. 224.

24. G i d d ing s I. C., J. Gas Chromatography, 1, 38 (1963).

25. S chw a rtz C. E., S m it h I. M., Ind. Eng. Chem., 45, 1209 (1953).

26. Шевелев Я. В., ЖФХ, 31, 2110 (1957).

27. Scheidegger A. E., The Physics of Flow through Porous Media, Mac Millan, N. Y., 1957 , p. 126.

28. Gi d d i ng s I. C., Fulle r E. N., J. Chromatography, 7, 255 (1962).

29. B a y e r E., H u pe K. P., M a c k H., Anal. Chem., 35, 492 (1963).

30. Frisone G., Worthington A. E., Gollob F., Nor em S. D., Am y I. W., Felt o n H. R., Gas Chromatography 1961, Academic Press, N. Y., 1962, pp. 528

31. Чуднов ский А. Ф., Теплообмен в дисперсных средах, Гостехиздат, М., 1954, стр. 372 .

32. Ч ернышев А. Б., Поме ранцев А. А., Ф ар беров И. Л., Докл. АН СССР, 56, 727 (1947).

33. Жуховицкий А. А., Т уркельт а уб Н. М., Газовая хроматография, Гостоптехиздат, М., 1962, стр. 376.

34. Collins R. E., Flow of Fluids through Porous Materials, Reinhold, N. Y., 1961, p. 51 .

35. Мулликандов, ЖТФ, 18, 1051 (1948).

36. Chilt o n H., Col bu r n P., Ind. Eng. Chem., 23, 910 (1931).

37. Fr is o ne G. J., J. Chromatography, 6, 97 (1961).

38. Л уйг а П. О., Л и п п м а Э Э. Т., Тр. Таллинск. политехн. ин-та, Сер. А, № 195, 1962, стр. 121 .

Ннститут кибернетики

Академии наук Эстонской ССР

Поступила в редакцию

29/VI 1964

\section{E. LIPPMAA, P. LUIGA}

\section{GAASI VOOLU EBAOHTLUSEST PREPARATIIVSETES GAASIKROMATOGRAAFILISTES KOLONNIDES}

Gaasikromatograafilise kolonni diameetri suurendamisega kaasnevat teoreetilise taldriku kōrguse suurenemist ei pōhjusta seinaefekt sel määral, kui seda on väitnud Golay jt., vaid on esmajoones tingitud gaasi voolu ebaühtlusest kogu kolonni ristlōike ulatuses. Kuna gaasi voolu kiirus on väikene ja ebaühtlus pole otseselt mõõdetav, siis määrati lahutatava aine frondi kuju preparatiivses kolonnis (diameeter 32 ja 75 mm) väikeste vesinik-leek-detektoritega ( 30 väiksemal ja 42 suuremal kolonnil). Selgus, et $0,25-0,5 \mathrm{~mm}$ jämeduse ja $30 \%$ dibutüülftalaadiga immutatud täidisega ning samasuguse immutamata täidisega täidetud kolonnis jōuab aine front kolonni keskosas ette. Aine «korgi» tagaserval esineb tunduv mahajäämus seinte ääres. Gaasi voolu ebaühtlasest kiirusest vähendatud kolonni lahutusvōimet saab parandada kolonni paigutatud tsentraalsete avadega 
vaheketaste abil. Nende mõju kirjeldas Frisone kui seinaefektist tingitud kausikujulisele, seina ääres etteliikuvale frondile perifeerse takistuse loomist. Niisugused kettad tõepoolest parandavad kolonni lahutusvōimet, kuid paremaid tulemusi võib saada tsentraalsete takistustega, mis jätavad kitsa perifeerse pilu. Mōlemat tüüpi kettad toimivad gaasi voolu segajatena, neist optimaalse määrab kolonni täitmise viis.

\section{E. LIPPMAA, P. LUIGA}

\section{A STUDY OF THE NONUNIFORMITY OF GAS FLOW IN LARGE-SCALE GAS CHROMATOGRAPHIC COLUMNS}

The increase in diameter of the height equivalent of a theoretical plate in large gas chromatographic columns is not due to the wall effect in such a degree as described by Golay and others, but rather to nonuniformity of the gas flow across the column. The gas flow velocity is small, and flow nonuniformity cannot be measured directly, and thereforé the form of the sample front in preparative scale columns (diameter 32 and $75 \mathrm{~mm}$ ), was measured with small hydrogen flame detectors ( 30 for the smaller, 42 for the bigger column). It appears that for our columns filled with 32-60 mesh firebrick impregnated with $30 \%$ dibutylphthalate, and in some runs without a liquid phase, the velocity of the sample front is highest in the central part of the column. There is considerable tailing along the walls. The adverse effect of nonuniform gas (and sample) velocity can be lessened with discs with central holes, designed to counteract the wall effect and the "inverted bowl"-shaped velocity profile with maximum velocity near the walls, as described by Frisone. Such discs do in fact increase column efficiency, yet even better results can be achieved with undersized washers without holes that leave a small peripheral slot along the walls. Both sorts of discs act mainly as mixing washers, and optimum design may: depend on the mode of column packing. 